\title{
Pacific
}

Journal of

Mathematics

\section{ASYMPTOTIC BEHAVIOR OF THE CURVATURE OF THE BERGMAN METRIC OF THE THIN DOMAINS}

KANG-TAE KIM 


\title{
ASYMPTOTIC BEHAVIOR OF THE CURVATURE OF THE BERGMAN METRIC OF THE THIN DOMAINS
}

\author{
KANG-TAE KIM
}

\begin{abstract}
The Riemann sectional curvature tensor of the Bergman metric of the domains that are 'thin' intersections of the (strongly) pseudoconvex domains in $C^{n}$ with $C^{2}$ boundaries, $n \geq 2$, is asymptotically equivalent to that of corresponding Siegel domains.
\end{abstract}

1. Thin domains in $\mathbf{C}^{n}$. Among the bounded domains of holomorphy in $\mathbf{C}^{n}$ with non-smooth boundaries, we consider the ones that can be obtained as the intersection of the two or more strongly pseudoconvex domains with $C^{2}$ boundaries. Precisely speaking, a typical such domain can be represented as:

$$
D=\left\{z \in \mathbf{C}^{n} \mid \rho_{1}(z)<0, \ldots, \rho_{k}(z)<0\right\}
$$

where:

(1) for each $j=1, \cdots, k$, the inequality $\rho_{j}(z)<0$ defines a strongly pseudoconvex domain with a $C^{2}$ boundary, and

(2) the defining functions satisfy that

$$
d \rho_{i_{1}} \wedge \cdots \wedge d \rho_{i_{q}} \neq 0 \quad(q \leq n)
$$

at every point where $\rho_{i_{1}}=\cdots=\rho_{i_{q}}=0$ for any appropriate $q$.

In this paper, we would like to give a description of the asymptotic behavior of the Riemann sectional curvature tensor of the Bergman metric ([1]) of such domains that are so-called 'thin'. (See Definition 2.) Our results are mainly focused on the case when the boundary point is singular, because the case when the target boundary point is regular has been well settled earlier by Klembeck [4], (see also [3]).

However, this paper is substantially different from the preceding works on asymptotic behavior of the Bergman curvature, since we treat the case when the boundary is singular. Lack of smoothness of the boundary rules out the possibility of using the well-known asymptotic expansion formula of the Bergman kernel function by $\mathrm{C}$. Feffermann [2]. But interestingly enough, even for the case of $C^{\infty}$ boundaries, 
our method gives a much easier and more intuitive proof than the original one in [4].

The analysis of asymptotic behavior of Bergman curvature explains the geometry of the Bergman metric near the boundary as well as the behavior of biholomorphic mappings. This also has applications to the study of automorphism groups and their orbits. However, we try not to include any details here.

We start with

Definition 1. Let $D$ be a domain in $\mathbf{C}^{n}$ and its boundary $\partial D$. By the singular locus of the boundary of $D$, we mean the set

$$
S_{\partial D}:=\{p \in \partial D \mid \partial D \text { is not smooth at } p\} .
$$

DEFINITION 2. Let the bounded strongly pseudoconvex domains $D_{1}, \cdots, D_{k}(2 \leq k)$ with $C^{2}$ smooth boundaries be defined by the defining functions $\rho_{1}, \ldots, \rho_{k}$, respectively. The intersection $D=\bigcap_{j=1}^{k} D_{j}$ is called thin, if

(1) $D$ is connected;

(2) $d \rho_{i_{1}} \wedge \cdots \wedge d \rho_{i_{s}} \neq 0$ at every point where $\rho_{i_{1}}=\cdots=\rho_{i_{s}}=0$;

(3) for each $p \in S_{\partial D}^{s}$ with $\rho_{i_{1}}(p)=\cdots=\rho_{i_{s}}=0$ and $\rho_{j} \neq 0$ for any $j \neq i_{m}, m=1, \ldots, s$, there is a one-to-one holomorphic mapping $g: D \rightarrow \mathscr{D}(s, n)$, where $\mathscr{D}(s, n)$ is one of the Siegel domains of the 2 nd kind introduced in $\S 2$ of this article, that has a continuous extension to the boundary such that $g(p)=0$.

(4) for each $t \in \partial D \backslash S_{\partial D}$, there is a one-to-one holomorphic mapping $h: D \rightarrow B^{n}$ that has a continuous extension to the boundary such that $h(t) \in \partial B^{n}$. Here, $B^{n}$ denotes the open unit ball in $\mathbf{C}^{n}$.

Note that any convex intersection is thin. We also point out that even if the last two conditions above may appear artificial and complicated, all these are satisfied obviously when the domain in consideration is indeed intuitively a thin intersection.

2. Asymptotic behavior of Bergman curvature. Since the boundaries of our domains described in the definition above are singular, we immediately face a difficulty due to the fact that there is no uniformity among the boundary points near any singular boundary point. So, before we state our main results, we would like to give a clear description for the asymptotic sequences in the following trichotomy:

Let $D$ be a thin bounded domain with a singular boundary. Let $p \in S_{\partial D}$. Let $\left\{p_{j}\right\} \subset D$ be a sequence converging in $\mathbf{C}^{n}$ to $p$.

(I) We say $p_{j}$ approaches $p$ non-tangentially to $\partial D$, if there exists 
a constant $C>0$ such that

$$
\frac{\operatorname{dis}\left(p_{j}, \partial D\right)}{\operatorname{dis}\left(p_{j}, p\right)} \geq C \text { for any } j \text {. }
$$

(II) We say $p_{j}$ approaches $p$ tangentially to $S_{\partial D}$, if there exists a constant $C>0$ such that

$$
\lim _{j \rightarrow \infty} \frac{\operatorname{dis}\left(p_{j}, \partial D\right)}{\operatorname{dis}\left(p_{j}, p\right)}=0 \quad \text { and } \quad \frac{\operatorname{dis}\left(p_{j}, \partial D\right)}{\operatorname{dis}\left(p_{j}, S_{\partial D}\right)} \geq C .
$$

(III) We say $p_{j}$ approaches $p$ tangentially to $\partial D$ away from $S_{\partial D}$, if

$$
\lim _{j \rightarrow \infty} \frac{\operatorname{dis}\left(p_{j}, \partial D\right)}{\operatorname{dis}\left(p_{j}, p\right)}=0 \text { and } \lim _{j \rightarrow \infty} \frac{\operatorname{dis}\left(p_{j}, \partial D\right)}{\operatorname{dis}\left(p_{j}, S_{\partial D}\right)}=0
$$

Some notations. Throughout this article, we note that

(1) $B^{k}(0, r)=$ the open ball in $\mathbf{C}^{k}$ centered at 0 with radius $r$.

(2) $B^{k}=B^{k}(0,1)$.

(3) $\mathscr{D}(s, n)=$ the Siegel domain in $\mathbf{C}^{n}$ of the second kind defined by the inequalities:

$$
\begin{gathered}
\operatorname{Im} z_{1}>\left|a_{1 s+1} z_{s+1}\right|^{2}+\cdots+\left|a_{1 n} z_{n}\right|^{2} \\
\vdots \\
\operatorname{Im} z_{s}>\left|a_{s s+1} z_{s+1}\right|^{2}+\cdots+\left|a_{s n} z_{n}\right|^{2}
\end{gathered}
$$

for some numbers $a_{j k}$, where $j=1, \ldots, s \leq n$ and $k=s+$ $1, \ldots, n$.

Main statements. Now, let $D$ be the thin intersection of the bounded strongly pseudoconvex domains $D_{1}, \ldots, D_{k}$, where $k \geq 2$. Let $p_{j} \in D$ form a sequence of points converging to a point $p \in \partial D$. Then we state three theorems which describe the asymptotic behavior of the sectional curvature tensor of the Bergman metric of the thin domains. One more thing to remark is that we need to be more specific about the convergence of two dimensional sections as well, especially because the limiting sectional curvature tensor is not constant in general. This consideration also appears in the statements of the main theorems. For convenience, we fix $p \in S_{\partial D}$ and regard $g$ as an inclusion map in Definition 2 of the preceding section. Moreover, for each integer $s$ and $j$, define map $L_{j}: \mathbf{C}^{n} \rightarrow \mathbf{C}^{n}$ by

$$
L_{s, j}\left(z_{1}, \ldots, z_{n}\right)=\left(\lambda_{j} z_{1}, \ldots, \lambda_{j} z_{s}, \sqrt{\lambda_{j}} z_{s+1}, \ldots, \sqrt{\lambda_{j}} z_{n}\right)
$$

where $\lambda_{j} \rightarrow \infty$ as $j \rightarrow \infty$. 
THeOREM 1 (Non-tangential behavior). If $p_{j} \rightarrow p \in S_{\partial D}$ nontangentially to $\partial D$. Then, as $j$ tends to infinity, there exists a subsequence of the sequence of the values at the 2-plane $P_{j} \subset T D_{p_{j}}$ of the Riemann sectional curvature tensor $\left\{R_{D}\left(P_{j}\right)\right\}$ of the Bergman metric of $D$ which converges to the sectional curvature tensor of the Bergman metric of one of the special Siegel domains $\mathscr{D}(s, n)$ evaluated at the 2-plane $\widehat{P}=\lim L_{s, j}\left(P_{j}\right)$ in the tangent space of $\mathscr{D}(s, n)$ at the point $\hat{p}=\left(w_{1}, \ldots, w_{n}\right)$ such that $\operatorname{Im} w_{1}>0, \ldots, \operatorname{Im} w_{s}>0, w_{s+1}=$ $\cdots=w_{n}=0$, where $s$ is the number of boundaries $\partial D_{j}$ 's which pass through the boundary point $p$ of $\partial D$. Moreover, $w_{1}, \ldots, w_{s}$ are canonically and explicitly determined by $\left\{p_{j}\right\}$.

The limits of the set here are taken in the sense of local Hausdorff set convergence.

Theorem 2 (Singular tangential behavior). If $p_{j} \rightarrow p \in S_{\partial D}$ tangentially to $S_{\partial D}$, by choosing a subsequence we may assume that $p_{j} \rightarrow p$ tangentially to a smooth (open) submanifold of $S_{\partial D}$ which is the intersection of $\partial D_{i_{1}}, \ldots, \partial D_{i_{r}}$. Then there exists a subsequence of the sequence $\left\{R_{D}\left(P_{j}\right)\right\}$ of the sectional curvature at the 2-plane $P_{j} \subset$ $T D_{p_{j}}$ which converges to the sectional curvature of the Bergman metric of the Siegel domain $\mathscr{D}(r, n)$ evaluated at the 2-plane $\lim L_{r, j}\left(P_{j}\right)$ in the tangent space of $\mathscr{D}(r, n)$ at $\left(w_{1}, \ldots, w_{n}\right)$, where $\operatorname{Im} w_{1}>$ $0, \ldots, \operatorname{Im} w_{r}>0, w_{s+1}=\cdots=w_{n}=0$. Again, $w_{1}, \ldots, w_{r}$ are canonically and explicitly determined by $\left\{p_{j}\right\}$.

Theorem 3 (Regular tangential behavior). If $p_{j} \rightarrow p \in S_{\partial D}$ tangentially to $\partial D$ away from $S_{\partial D}$ or if $p_{j} \rightarrow p \in \partial D \backslash S_{\partial D}$, then the sequence of the sectional curvature tensor $\left\{R_{D}\left(p_{j}\right)\right\}$ converges to the sectional curvature of the Bergman metric of the open unit ball $B^{n}$ in $\mathrm{C}^{n}$. In this case, the sequence $L_{1, j}$ is in effect.

Combining the theorems in [4], [6], [7], [10], [11] and the theorems above, one can obtain the following information on the orbit of any point of a thin domain under the action of its automorphism group.

COROllary. Let $D$ be a thin domain in $\mathbf{C}^{n}$ with a piecewise smooth but not entirely smooth boundary. Suppose that its automorphism group is non-compact. Then any orbit of a point under the action of the automorphism group accumulates at a singular boundary points either 
nontangentially to the boundary of $D$ or tangentially to the singular locus of the boundary of $D$.

Theorems in the above can serve as an effective tool to compute the Bergman curvature of the thin domains in general, if more about the sectional curvature of the Bergman metric of the Siegel domains are known. At least, in the special cases $n \leq 4$ or $s \leq 2$, the curvature tensor of the Bergman metric of special Siegel domains of second kind such as $\mathscr{D}(s, n) \subset \mathbf{C}^{n}$ is completely understood as in $\S 4$ of this paper. So these theorems indeed provide a complete description of the asymptotic behavior of the sectional curvature of the Bergman metric of such thin domains.

Moreover, essentially the same technique will yield a somewhat simpler proof of the theorem of Klembeck [4]. Of course, general strongly pseudoconvex domains are not necessarily thin. However, if the boundary is $C^{\infty}$ smooth, it is known that one can localize the problem of Bergman curvature (cf. [2], [5] et al.). Hence, we can work on the thin piece of the domain which can be treated via the method of this paper. So we obtain an alternative proof of

THEOREM (Klembeck). Let $\Omega$ be a bounded strongly pseudoconvex domain in $\mathbf{C}^{n}$ with a $C^{\infty}$ smooth boundary. Let $\left\{p_{j}\right\} \subset \Omega$ be a sequence accumulating to a boundary point. Then the sequence of the values at $p_{j}$ of the Riemann sectional curvature tensor of the Bergman metric of $\Omega$ converges to that of the open unit ball in $\mathbf{C}^{n}$.

The author would like to express his gratitude to C.K. Cheung, R.E. Greene and $\mathrm{H}$. Wu for their interest and comments.

3. Proof of the theorems. Our proof depends partly upon the interior stability of the Bergman kernel function first proven by I. Ramadanov [9]. So, we begin our proof with

Interior stability of the Bergman kernel. The classical arguments in [9] easily prove the following:

Proposition 1 (Ramadanov). Let $D$ be a bounded domain in $\mathbf{C}^{n}$ containing the origin. Let $\left\{D_{n}\right\}$ be a sequence of bounded domains in $\mathbf{C}^{n}$ whose closures converge to the closure of the bounded domain $D$ in the sense of Hausdorff set convergence in such a way that for any $\varepsilon>0$ there exists $N>0$ such that

$$
(1-\varepsilon) D \subset D_{n} \subset(1+\varepsilon) D \quad \text { for any } n \geq N \text {. }
$$


Then, on each compact subset of D, the sequence of the Bergman kernel functions $K_{D_{n}}$ of $D_{n}$ converges uniformly to the Bergman kernel function $K_{D}$ of $D$. In particular, by Cauchy estimates, it follows that $K_{D_{n}}$ converges to $K_{D}$ in the $C^{\infty}$ sense on any fixed compact subset of $D$.

It may be important to notice that no extra regularity assumption on the boundary of $D$ is necessary in the above. The proof of the proposition is straightforward, and hence is omitted.

Asymptotic Bergman curvature. Let $D$ be the thin intersection of strongly pseudoconvex domains $D_{1}, \ldots, D_{k}$, where $k \geq 2$, be with $C^{2}$ defining functions $\rho_{1}, \ldots, \rho_{k}$ respectively. To investigate the asymptotic behavior of the Riemann sectional curvature tensor of the Bergman metric of $D$, we deal with the three different types of asymptotic sequences separately.

Proof of Theorem 1. Let $\left\{p_{j}\right\} \subset D$ be a sequence that approaches $p \in S_{\partial D}$ in Euclidean distance non-tangentially to $\partial D$.

Without loss of generality, we may assume that

$$
\rho_{1}(p)=\cdots=\rho_{s}(p)=0
$$

and

$$
\rho_{s+1}(p)<0, \ldots, \rho_{n}(p)<0 .
$$

Since $D$ is a thin intersection and $p \in S_{\partial D}$, we have $2 \leq s \leq n$. Using a complex linear change of coordinates if necessary, we may also assume that $p$ is the origin of $\mathbf{C}^{n}$, that $p_{j} / \operatorname{dis}\left(p, p_{j}\right)$ converges to a point in $C^{n}$, and that near $p=0, D$ is defined by the inequalities

$$
\begin{aligned}
\operatorname{Im} z_{1} & >\Phi_{1}\left(z_{1}, \ldots, z_{n}\right) \\
\vdots & \\
\operatorname{Im} z_{s} & >\Phi_{s}\left(z_{1}, \ldots, z_{n}\right)
\end{aligned}
$$

where:

(1) $\Phi_{k}(0)=0$, for any $k=1, \ldots, s$,

(2) $\left.\left(\partial \Phi_{k} / \partial z_{l}\right)\right|_{0}=\left.\left(\partial \Phi_{k} / \partial \bar{z}_{l}\right)\right|_{0}=0$ for all $k=1, \ldots, s$ and all $l=1, \ldots, n$, and

(3) the Levi form of $\Phi_{k}$ is positive definite at every boundary point of $D$ near 0 for each $k=1, \ldots, s$. 
Let $\delta_{j}=\operatorname{dis}\left(p, p_{j}\right)$ and let $\lambda_{j}=1 / \delta_{j}$ for each natural number $j$. Also define the linear map $L_{j}: \mathbf{C}^{n} \rightarrow \mathbf{C}^{n}$ by

$$
L_{j}\left(z_{1}, \ldots, z_{n}\right)=\left(\lambda_{j} z_{1}, \ldots, \lambda_{j} z_{s}, \sqrt{\lambda_{j}} z_{s+1}, \ldots, \sqrt{\lambda_{j}} z_{n}\right)
$$

for any $\left(z_{1}, \ldots, z_{n}\right) \in \mathbf{C}^{n}$.

Then observe that for any $R>0$ there exists $N>0$ such that $L_{j}(D) \cap B^{n}(0, R)$ consists of all the points $\left(z_{1}, \ldots, z_{n}\right) \in B^{n}(0, R)$ satisfying the inequalities

$$
\begin{gathered}
\operatorname{Im} \delta_{j} z_{1}>\Phi_{1}\left(\delta_{j} z_{1}, \ldots, \delta_{j} z_{s}, \sqrt{\delta_{j}} z_{s+1}, \ldots, \sqrt{\delta_{j}} z_{n}\right) \\
\vdots \\
\operatorname{Im} \delta_{j} z_{s}>\Phi_{s}\left(\delta_{j} z_{1}, \ldots, \delta_{j} z_{s}, \sqrt{\delta_{j}} z_{s+1}, \ldots, \sqrt{\delta_{j}} z_{n}\right) .
\end{gathered}
$$

Therefore, for each $R>0$, the Hausdorff limit of the sequence $L_{j}(D) \cap B^{n}(0, R)$ consists of all the points $z \in B^{n}(0, R)$ satisfying

$$
\begin{gathered}
\operatorname{Im} z_{1}>\widetilde{\Phi}_{1}\left(0, \ldots, 0, z_{s+1}, \ldots, z_{n}\right) \\
\vdots \\
\operatorname{Im} z_{s}>\widetilde{\Phi}_{s}\left(0, \ldots, 0, z_{s+1}, \ldots, z_{n}\right)
\end{gathered}
$$

where $\widetilde{\Phi}_{l}$ denotes the second order homogeneous polynomial in $z, \bar{z}$, where $z=\left(z_{1}, \ldots, z_{n}\right)$, in the Taylor expansion of $\rho_{l}$ at $p=0$ for each $l=1, \ldots, s$. Moreover, note that the Levi form of $\widetilde{\Phi}_{l}\left(0, \ldots, 0, z_{s+1}, \ldots, z_{n}\right)$ with respect to $\left(z_{s+1}, \ldots, z_{n}\right)$ is positive definite Hermitian. Therefore, in the local Hausdorff distance convergence, the limit $\widehat{D}$ of the sequence $\left\{L_{j}(D)\right\}$ is the domain in $\mathbf{C}^{n}$ defined by the inequalities

$$
\begin{gathered}
\operatorname{Im} z_{1}>F_{1}\left(z_{s+1}, \ldots, z_{n}\right) \\
\vdots \\
\operatorname{Im} z_{s}>F_{s}\left(z_{s+1}, \ldots, z_{n}\right)
\end{gathered}
$$

where $F_{l}\left(z_{s+1}, \ldots, z_{n}\right)=\widetilde{\Phi}_{l}\left(0, \ldots, 0, z_{s+1}, \ldots, z_{n}\right)$ for each $l=$ $1, \ldots, s$. By an obvious (global) holomorphic coordinate change, it is easy to see that $\widehat{D}$ is biholomorphic to the domain $\mathscr{D}(s, n)$ defined by

$$
\begin{gathered}
\operatorname{Im} z_{1}>\left|a_{1 s+1} z_{s+1}\right|^{2}+\cdots+\left|a_{1 n} z_{n}\right|^{2} \\
\vdots \\
\operatorname{Im} z_{s}>\left|a_{s s+1} z_{s+1}\right|^{2}+\cdots+\left|a_{s n} z_{n}\right|^{2} .
\end{gathered}
$$


According to [8], $\mathscr{D}(s, n)$ is biholomorphic to a bounded domain in $\mathbf{C}^{n}$ via a linear fractional transformation. In fact, in our special case, we can use the condition (3) of Definition 2 in $\S 1$ of this paper, we can find a linear fractional transformation $F: \mathbf{C}^{n} \rightarrow \mathbf{C}^{n}$ which is well defined on $L_{j}(D)$ for any $j$ such that $F\left(L_{j}(D)\right)$ is bounded for any $j$.

Since $F$ maps the point at infinity to a single point, in our arguments it is not hard to see that for any $\varepsilon>0$ there exists a positive integer $j_{0}$ such that

$$
(1-\varepsilon) F(\mathscr{D}(s, n)) \subseteq F\left(L_{j}(D)\right) \subseteq(1+\varepsilon) F(\mathscr{D}(s, n))
$$

for all $j \geq j_{0}$. Moreover, the sequence $\left\{L_{j}\left(p_{j}\right)\right\}$ is contained in a fixed compact set contained in an open subset of

$$
F(\mathscr{D}(s, n)) \cap\left(\bigcap_{j=1}^{\infty} F\left(L_{j}(D)\right)\right) .
$$

Let $X_{j}, Y_{j} \in T D_{p_{j}}$. Then notice that by extracting a subsequence, the sequence $\left\{P_{j}\right\}$ of 2-planes spanned by $\left\{L_{j} X_{j}, L_{j} Y_{j}\right\}$ converges to a 2-plane $\lim P_{j}$ in $T \mathscr{D}(s, n)_{\lim L_{j} p_{j}}$ in local Hausdorff distance. Hence, by Proposition 1, the Riemann sectional curvature tensor $R_{D}$ of the Bergman metric of $D$ satisfies

$$
\begin{aligned}
\lim _{j \rightarrow \infty} R_{D, p_{j}}\left(P_{j}\right) & =\lim _{j \rightarrow \infty} R_{L_{j}(D), L_{j}\left(p_{j}\right)}\left(L_{j}\left(P_{j}\right)\right) \\
& =R_{\mathscr{D}(s, n), \hat{p}}\left(\lim L_{j}\left(P_{j}\right)\right)
\end{aligned}
$$

where $\hat{p}=\lim _{j \rightarrow \infty} L_{j}\left(p_{j}\right)=\left(w_{1}, \ldots, w_{n}\right)$ with $\operatorname{Im} w_{1}>0, \ldots$, $\operatorname{Im} w_{s}>0, w_{s+1}=\cdots=w_{n}=0$. So Theorem 1 follows.

Proof of Theorem 2. Let $p_{j} \rightarrow p \in S_{\partial D}$ tangentially to $S_{\partial D}$.

We may assume without loss of generality that $p_{j} \rightarrow p$ (by choosing a subsequence if necessary) tangentially to a smooth open submanifold $N$ of $S_{\partial D}$ which is contained in the variety defined by $\rho_{1}=\cdots=$ $\rho_{r}=0$. Clearly, $\rho_{1}(p)=\cdots=\rho_{r}(p)=0$ by continuity. Now choose, for each $j=1,2, \ldots, q_{j} \in N$ such that $\operatorname{dis}\left(p_{j}, q_{j}\right)=\operatorname{dis}\left(p_{j}, S_{\partial D}\right)$.

Again, for each $j$, by a (global) complex linear change, say $S \in$ $G L(n, \mathbf{C})$, of coordinates, near $q_{j}$, the inequalities

$$
\rho_{1}(z)<0, \ldots, \rho_{r}(z)<0
$$


can be re-written simultaneously as

$$
\begin{aligned}
\operatorname{Im} z_{1} & >\Psi_{1}\left(z_{1}, \ldots, z_{n}\right) \\
\vdots & \\
\operatorname{Im} z_{r} & >\Psi_{r}\left(z_{1}, \ldots, z_{n}\right)
\end{aligned}
$$

where:

(1) $q_{j}=0$,

(2) $\Psi_{l}(0)=0,\left.\left(\partial \Psi_{l} / \partial z_{k}\right)\right|_{0}=\left.\left(\partial \Psi_{l} / \partial \bar{z}_{k}\right)\right|_{0}=0$, for all $l=$ $1, \ldots, r, k=1, \ldots, n$, and

(3) the Levi form of $\Psi_{k}$ is positive definite at every boundary point of $D$ near 0 .

Notice that, even if $\Psi_{l}$ 's depend upon $j$, the dependence is uniform in the $C^{2}$ sense as $j \rightarrow \infty$. So throughout our arguments, whenever we ignore $j$-dependence, we appeal to the uniform $C^{2}$ dependence on $j$ unless otherwise mentioned.

Let $\delta_{j}:=\operatorname{dis}\left(p_{j}, q_{j}\right)$ and $\lambda_{j}=1 / \delta_{j}$, for each $j=1,2, \ldots$ Then first note that (by the law of cosines and smoothness of $\rho_{l}$ 's)

$$
\lim _{j \rightarrow \infty} \lambda_{j} \operatorname{dis}\left(p_{j}, p\right)=\infty .
$$

Let us identify $S(D)$ with $D$. Furthermore, we claim that, for $p=$ $\left(p_{1}, \ldots, p_{n}\right)$ and $q_{j}=(0, \ldots, 0)$,

$$
\lim _{j \rightarrow \infty}\left(\lambda_{j} p_{1}, \ldots, \lambda_{j} p_{r}, \sqrt{\lambda_{j}} p_{r+1}, \ldots, \sqrt{\lambda_{j}} p_{n}\right)=\infty .
$$

This follows from the fact that under the effect of repeated linear scaling by

$$
L_{j}\left(z_{1}, \ldots, z_{n}\right):=\left(\lambda_{j} z_{1}, \ldots, \lambda_{j} z_{r}, \sqrt{\lambda_{j}} z_{r+1}, \ldots, \sqrt{\lambda_{j}} z_{n}\right)
$$

the points $\left(c_{1}, \ldots, c_{n}\right)$ on the boundary $\partial L_{j}(D)$ of $L_{j}(D)$ satisfy the inequalities

$$
\operatorname{Im} c_{l}=\Psi_{l}\left(c_{r+1}, \ldots, c_{n}\right), \quad \text { for all } l=1, \ldots, r,
$$

modulo some insignificant error (e.g. see the scaling in the proof of Theorem 1). Hence, $(\dagger)$ implies that $\left(\lambda_{1} p_{1}, \ldots, \lambda_{r} p_{r}, \sqrt{\lambda_{j}} p_{r+1}, \ldots\right.$, $\left.\sqrt{\lambda_{j}} p_{n}\right) \rightarrow \infty$ as $j \rightarrow \infty$.

In addition, we note the obvious fact that, with $q_{j}=0$,

$(\dagger \dagger \dagger) \lim _{j \rightarrow \infty} L_{j}(t)=\infty$ for any $t$ satisfying $\rho_{1}(t)^{2}+\cdots+\rho_{r}(t)^{2} \neq 0$. 
By $(\dagger),(\dagger \dagger)$ and $(\dagger \dagger \dagger)$, for any $R>0$, there exists a positive integer $j_{0}$ such that $L_{j}(D) \cap B^{n}(0, R)$ consists of all the points $\left(z_{1}, \ldots, z_{n}\right)$ $\in B^{n}(0, R)$ satisfying the inequalities

$$
\begin{gathered}
\operatorname{Im} \delta_{j} z_{1}>\Psi_{1}\left(\delta_{j} z_{1}, \ldots, \delta_{j} z_{r}, \sqrt{\delta_{j}} z_{r+1}, \ldots, \sqrt{\delta_{j}} z_{n}\right) \\
\vdots \\
\operatorname{Im} \delta_{j} z_{r}>\Psi_{r}\left(\delta_{j} z_{1}, \ldots, \delta_{j} z_{r}, \sqrt{\delta_{j}} z_{r+1}, \ldots, \sqrt{\delta_{j}} z_{n}\right) .
\end{gathered}
$$

Then the rest of the proof of this case is similar to that of Theorem 1. This completes the proof of Theorem 2 .

Proof of Theorem 3. Assume that $p_{j} \rightarrow p \in S_{\partial D}$ tangentially to $\partial D$ away from $S_{\partial D}$, or $p_{j} \rightarrow p \in \partial D \backslash S_{\partial D}$.

It turns out now that this is the easiest to prove among all three theorems. All the arguments will be similar to the previous cases except that the local Hausdorff limit of $\left\{L_{j}(D)\right\}$ is defined by only one inequality

$$
\operatorname{Im} z_{1}>\left|b_{2} z_{2}\right|^{2}+\cdots+\left|b_{n} z_{n}\right|^{2},
$$

where $L_{j}(z)=\left(\lambda_{j} z_{1}, \sqrt{\lambda_{j}} z_{2}, \ldots, \sqrt{\lambda_{j}} z_{n}\right)$. It is well-known that such a domain is biholomorphic to the unit ball in $\mathbf{C}^{n}$. Consequently, using condition (4) of Definition 2 and the same stability arguments, we obtain the desired conclusion.

4. Curvature of certain Siegel domains. In this section, we compute the curvature tensor of the Bergman metric of special types of Siegel domains $\mathscr{D}(s, n)$ for $n \leq 4$ and for $s \leq 2$. In fact, all the essential techniques applicable to $\mathscr{D}(n-2, n)$ can be seen in the computation of $\mathscr{D}(2,4)$. Hence we only compute

Bergman curvature of $D(2,4)$. Up to biholomorphic equivalences, $\mathscr{D}(2,4)$ is defined by the two inequalities

$$
\begin{aligned}
& \operatorname{Im} z_{1}>\left|z_{3}\right|^{2}+\left|z_{4}\right|^{2} \\
& \operatorname{Im} z_{2}>\left|z_{3}\right|^{2}+\left|c z_{4}\right|^{2}
\end{aligned}
$$

for some $c>0$.

To compute the Bergman curvature of this domain, we consider the domain $\mathscr{E} \subset \mathbf{C}^{6}$ defined by

$$
\begin{aligned}
& \operatorname{Im} \zeta_{1}>\left|\zeta_{2}\right|^{2}+\left|\zeta_{3}\right|^{2} \\
& \operatorname{Im} \zeta_{4}>\left|\zeta_{5}\right|^{2}+\left|c \zeta_{6}\right|^{2} .
\end{aligned}
$$


It is well-known that $\mathscr{E}$ is biholomorphic to $B^{3} \times B^{3}$, by a standard linear fractional transformation, say $\varphi: \mathscr{E} \rightarrow B^{3} \times B^{3}$.

Notice then that the map $f: \mathscr{D}(2,4) \rightarrow \mathscr{E}$ defined by

$$
f\left(z_{1}, z_{2}, z_{3}, z_{4}\right)=\left(z_{1}, z_{3}, z_{4}, z_{2}, z_{3}, z_{4}\right)
$$

is a proper holomorphic embedding. Moreover, $M:=f(\mathscr{D}(2,4))$ is in fact the fixed point set of the involutive automorphism $g$ of $\mathscr{E}$ defined by

$$
g\left(\zeta_{1}, \zeta_{2}, \zeta_{3}, \zeta_{4}, \zeta_{5}, \zeta_{6}\right)=\left(\zeta_{1}, \zeta_{5}, \zeta_{6}, \zeta_{4}, \zeta_{2}, \zeta_{3}\right)
$$

Hence, $M$ is totally geodesic in $\mathscr{E}$ with respect to the Bergman metric. Consequently, for any tangent vectors $X_{1}, X_{2}, X_{3}, X_{4}$ to the domain $\mathscr{D}(2,4)$ at $p \in \mathscr{D}(2,4)$, the curvature tensors $R_{\mathscr{D}}=R_{\mathscr{D}(2,4)}$ and $R_{B^{3} \times B^{3}}$ of the Bergman metrics of domains $\mathscr{D}(2,4)$ and $B^{3} \times B^{3}$, respectively, satisfy the relation

$$
\left.R_{\mathscr{D}}\left(X_{1}, X_{2}, X_{3}, X_{4}\right)\right|_{p}=\left.R_{B^{3} \times B^{3}}\left(\varphi_{*} f_{*} X_{1}, \ldots, \varphi_{*} f_{*} X_{4}\right)\right|_{\varphi \circ f(p)}
$$

which gives explicitly the values of the Bergman curvature desired.

In particular, together with Theorems 1, 2 and 3, we get the following result in contrast to [12].

Corollary. Let $D$ be an intersection of two balls in $\mathbf{C}^{2}$. Let $\left\{p_{j}\right\} \subset D$ be a sequence such that $p_{j} \rightarrow p \in S_{\partial D}$, as $j \rightarrow \infty$. Then the sequence of the 4-linear forms which are the Bergman curvature tensor of $D$ evaluated at $p_{j}, j=1,2, \ldots$ converges to the curvature tensor (evaluated at any point) of the Bergman metric of

(1) the bidisk $\Delta \times \Delta \subset \mathbf{C}^{2}$ if $p_{j} \rightarrow p$ nontangentially to $\partial D$ or if $p_{j} \rightarrow p$ tangentially to $S_{\partial D}$; and

(2) the unit ball $B^{2} \subset \mathbf{C}^{2}$ if $p_{j} \rightarrow p$ tangentially to $\partial D \backslash S_{\partial D}$.

Final Remark. The same method we used in this paper seems to be effective as well in computing the asymptotic behavior of the Bergman curvature of certain weakly pseudoconvex domains in $\mathbf{C}^{n}$ with piecewise $C^{2}$ Leviflat boundaries in some restricted cases. However, we do not include any details here. 


\section{REFERENCES}

[1] S. Bergman, The kernel function and conformal mappings, Math. Surveys, vol. 5, Amer. Math. Soc., Providence, R.I., 1950.

[2] C. Feffermann, The Bergman kernel and biholomorphic mappings of pseudoconvex domains, Invent. Math., 26 (1974), 1-65.

[3] R. Greene and S. Krantz, Stability properties of the Bergman kernel and curvature properties of bounded domains, Recent Developments in Several Complex Variables (J. E. Fornaess, ed.) (1981), 179-198, Ann. of Math. Studies, Princeton Univ. Press, no. 100.

[4] P. Klembeck, Kähler metrics of negative curvature, the Bergman metric near the boundary and the Kobayashi metric on smooth bounded strictly pseudoconvex sets, Indiana Univ. Math. J., 27 (1978), 275-282.

[5] J. J. Kohn, Regularity at the boundary of the $\bar{\partial}$ Neumann problem, Proc. Nat. Acad. Sci. U.S.A., 49 (1963), 206-213.

[6] Q. K. Lu, On Kähler manifolds with constant curvature, Acta Math. Sinica, 16 (1966), 269-281.

[7] S. Pinčuk, Holomorphic inequivalences of some classes of domains in $\mathbf{C}^{n}$, Math. USSR Sbornik, 39 (1981), 61-86.

[8] I. Pyatetskii-Shapiro, Automorphic functions and the geometry of classical domains, Gordon and Breach Sci. Pub., New York, London, Paris, 1969.

[9] I. Ramadanov, Sur une propriété de la fonction de Bergman, C.R. Acad. Bulgare Sci., 20 (1967), 759-762.

[10] J. P. Rosay, Sur une caracterization de la boule parmi les domaines de $\mathbf{C}^{n}$ par son group d'automorphismes, Ann. Inst. Fourier (Grenoble), 29 (1979), 91-97.

[11] B. Wong, Characterization of the unit ball in $\mathbf{C}^{n}$ by its automorphism group, Invent. Math., 41 (1977), 253-257.

[12] H. Wu, Old and new invariant metrics on complex manifolds, (preprint) (1989).

Received January 22, 1991 and in revised form April 26, 1991.

BROWN UNIVERSITY

Providence, RI 02912 


\section{PACIFIC JOURNAL OF MATHEMATICS EDITORS}

V. S. VARADARAJAN (Managing Editor)

University of California

Los Angeles, CA 90024-1555

vsv@math.ucla.edu

Herbert Clemens

University of Utah

Salt Lake City, UT 84112

clemens@math.utah.edu

F. Michael Christ

University of California

Los Angeles, CA 90024-1555

christ@math.ucla.edu

THOMAS ENRIGHT

University of California, San Diego

La Jolla, CA 92093

tenright@ucsd.edu
Nicholas ERcolani

University of Arizona

Tucson, AZ 85721

ercolani@math.arizona.edu

R. FINN

Stanford University

Stanford, CA 94305

finn@gauss.stanford.edu

VAUGHAN F. R. JoNES

University of California

Berkeley, CA 94720

vfr@math.berkeley.edu

STEVEN KeRCKHOFF

Stanford University

Stanford, CA 94305

spk@gauss.stanford.edu
C. C. MOORE

University of California

Berkeley, CA 94720

Martin ScharlemanN

University of California

Santa Barbara, CA 93106

mgscharl@henri.ucsb.edu

Harold Stark

University of California, San Diego

La Jolla, CA 92093

\begin{tabular}{|c|c|c|c|c|}
\hline \multicolumn{5}{|c|}{ ASSOCIATE EDITORS } \\
\hline R. ARENS & $\begin{array}{l}\text { E. F. BECKENBACH } \\
(1906-1982)\end{array}$ & B. H. NeumanN & $\begin{array}{c}\text { F. WoLF } \\
(1904-1989)\end{array}$ & K. YoshidA \\
\hline \multicolumn{5}{|c|}{ SUPPORTING INSTITUTIONS } \\
\hline \multicolumn{2}{|c|}{ UNIVERSITY OF ARIZONA } & \multicolumn{3}{|c|}{ UNIVERSITY OF OREGON } \\
\hline \multicolumn{2}{|c|}{ UNIVERSITY OF BRITISH COLUMB } & \multicolumn{3}{|c|}{$\begin{array}{l}\text { UNIVERSITY OF SOUTHERN CALIFORNI } \\
\text { STANFORD UNIVERSITY }\end{array}$} \\
\hline \multicolumn{2}{|c|}{ CALIFORNIA INSTITUTE OF TECHNOLOGY } & \multirow{2}{*}{\multicolumn{3}{|c|}{$\begin{array}{l}\text { STANFORD UNIVERSITY } \\
\text { UNIVERSITY OF HAWAII }\end{array}$}} \\
\hline \multicolumn{2}{|c|}{ UNIVERSITY OF CALIFORNIA } & & & \\
\hline \multicolumn{2}{|c|}{ MONTANA STATE UNIVERSITY } & \multicolumn{3}{|c|}{ UNIVERSITY OF TOKYO } \\
\hline \multicolumn{2}{|c|}{ UNIVERSITY OF NEVADA, RENO } & \multicolumn{3}{|c|}{ UNIVERSITY OF UTAH } \\
\hline \multicolumn{2}{|c|}{ NEW MEXICO STATE UNIVERSITY } & \multicolumn{3}{|c|}{ WASHINGTON STATE UNIVERSITY } \\
\hline \multicolumn{2}{|c|}{ OREGON STATE UNIVERSITY } & \multicolumn{3}{|c|}{ UNIVERSITY OF WASHINGTON } \\
\hline
\end{tabular}




\section{PACIFIC JOURNAL OF MATHEMATICS}

Volume $155 \quad$ No. $1 \quad$ September 1992

Characterization of modular correspondences by geometric properties 1 Allan Russell AdLer

Representations of convex nondentable sets

SPIROS ARGYROS and IRENE DELIYANNI

Isomorphisms of spaces of continuous affine functions

CHO-Ho CHU and HENRY BRUCE COHEN

Universal classes of Orlicz function spaces

FRANCISCO LUIS HERNÁNDEZ RODRÍGUEZ and CESAR RUIZ

Asymptotic behavior of the curvature of the Bergman metric of the thin 99 domains

KANG-TAE KIM

Quadratic central polynomials with derivation and involution

CHARLES PHILIP LANSKI

Nonsplit ring spectra and products of $\beta$-elements in the stable homotopy of Moore spaces

\section{JIN KUN LIN}

Orientation and string structures on loop space

DENNIS MCLAUGHLIN

Homomorphisms of Bunce-Deddens algebras

\section{CORNEL PASNICU}

Certain $C^{*}$-algebras with real rank zero and their corona and multiplier 169 algebras. Part I

SHUANG ZHANG

Correction to: "On the density of twistor elementary states"

Michael G. Eastwood and A. M. Pilato 